\title{
Modeling Chemical Reactions in Modelica By Use of Chemo-bonds
}

\author{
François E. Cellier \\ ETH Zürich \\ Switzerland \\ FCellier@Inf.ETHZ.CH
}

\author{
Jürgen Greifeneder \\ ABB AG, Corporate Research Center \\ Germany \\ Juergen.Greifeneder@,DE.ABB.Com
}

\begin{abstract}
This paper describes a new methodology for modeling and simulating chemical reaction systems using vectors of chemo-bonds, called multi-chemo-bonds. Chemical reactions are usually described through mass flows alone. Yet in reality, they are convective flows, as the reactants carry their volume and heat with them in the reactions. Each combined mass/volume/heat flow can be described by a chemo-bond. The combination of all such flows can be described by a vector of chemo-bonds, i.e., a multichemo-bond.
\end{abstract}

Keywords: object-oriented modeling of chemical reactions; chemo-bonds; thermo-bonds; multichemo-bonds; convective flows

\section{Introduction}

Traditionally, chemical reaction systems are described as pure mass flow systems. There is no need to consider the energy flows as well, as long as the thermo-dynamical properties of the reaction system can be ignored. As chemical reactions are characterized by capacitive storage only, i.e., they don't feature inductive storage, the mass balance and energy balance equations are decoupled from each other [2].

A first attempt of describing the thermodynamics of chemical reaction systems in a systematic way was reported in [5]. Unfortunately, the primary author of that paper, Aharon Katzir-Katchalsky, died prematurely during an attack by Palestinians on the airport of Tel-Aviv. After his death, this line of research stopped for several years.
His research was continued 12 years later by a student of one of the authors, Michael Amrhein, who described for the first time a chemical reaction system by means of bond graphs [1].

In the remainder of this paper, we start out with modeling the molar flows (Section 2) and put them together in a multi-bond representation (Section 3). In Section 4, the corresponding volume and entropy flows will be added to the molar flows resulting in a chemo-bond model. Section 5 is similar to Section 3 and introduces multi-chemo-bonds. Section 6 finally offers some conclusions.

\section{The basic model}

In Amrhein's work [1], the mass flow variable,

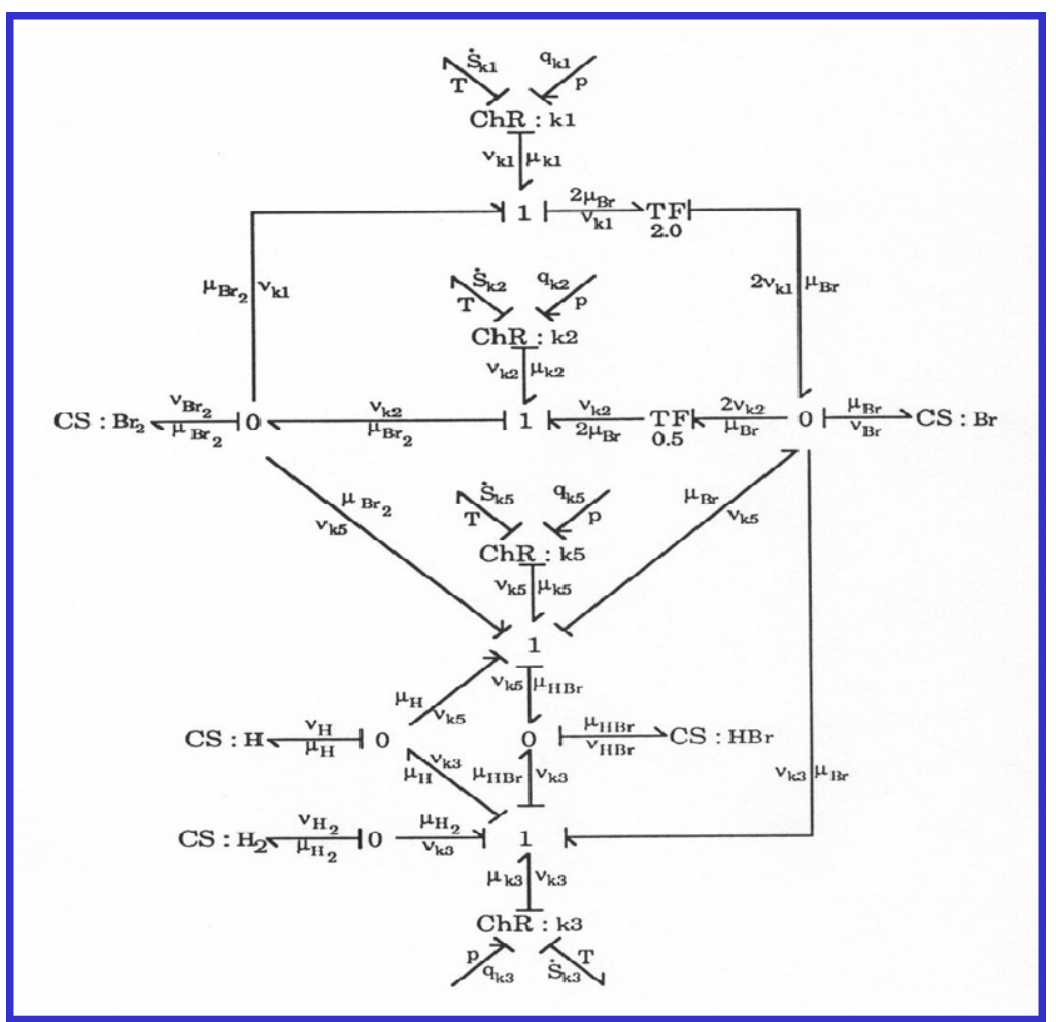

Fig.1: Chemical reaction bond graph 
i.e., the molar flow of a reactant in a reaction, is accompanied by a potential variable, the chemical potential, such that the product of the two variables denotes energy flow. Amrhein's bond graph simulated simultaneously the mass flow and the massenergy flow through the reaction system. He modeled a hydrogen-bromine reaction to be simulated under different operating conditions. The simplest experimental setup freezes the temperature and the pressure, i.e., the chemical reaction is simulated under isothermal and isobaric operating conditions. Amrhein's model is shown in Fig.1.

Amrhein developed his new modeling methodology years before Modelica was created, and he coded his models in the old Dymola language before a graphical interface had become available for Dymola. Hence the bond graph of Fig. 1 is not a code, but only a picture that was drawn manually to make the code better understandable.

The CS-elements represent the (capacitive) storage of the five reactants, whereas the ChR-elements represent the five individual step reactions. They are connected by a network of bonds, junctions, and transformers representing the chemical reaction network.

Yet, the graph shown in Fig.1 is far from complete. First, it only shows four ChR-elements, although the hydrogen-bromine reaction exhibits five individual step reactions, namely:

$$
\begin{aligned}
& \mathrm{Br}_{2} \underset{k_{2}}{\stackrel{k_{1}}{\rightleftharpoons}} 2 \mathrm{Br} \bullet \\
& \mathrm{Br} r^{\bullet}+\mathrm{H}_{2} \underset{k_{4}}{\stackrel{k_{3}}{\rightleftarrows}} \mathrm{HBr}+H^{\bullet} \\
& \mathrm{Br}_{2}+\mathrm{H}^{\bullet} \stackrel{k_{5}}{\rightarrow} \mathrm{HBr}+\mathrm{Br}^{\bullet}
\end{aligned}
$$

Reaction \#4, the least important of the five step reactions, was generously left out of the graph to keep the graph planar. Second, the chemical reactors, i.e., the ChR-elements, require state information that is being computed by the CS-elements. Hence there need to be added signal paths between the CSelements and the ChR-elements that were also left out for enhanced readability.

A completed graph of Amrhein's model, now coded in a current version of Dymola using BondLib [4] is shown in Fig.2. The grey lines are used to connect bonds with junctions and vice-versa, i.e., they represent the same energy flows as the bonds connected to them. The blue lines represent information flows. 0 and 1 represent junctions, and the half-arrows represent bonds.

The model of Fig. 2 can be simulated in Dymola without any problems, and for a temperature of $T=$ $800 \mathrm{~K}$ and a pressure of $p=102 \mathrm{kPa}$, we obtain the trajectories shown in Fig.3.

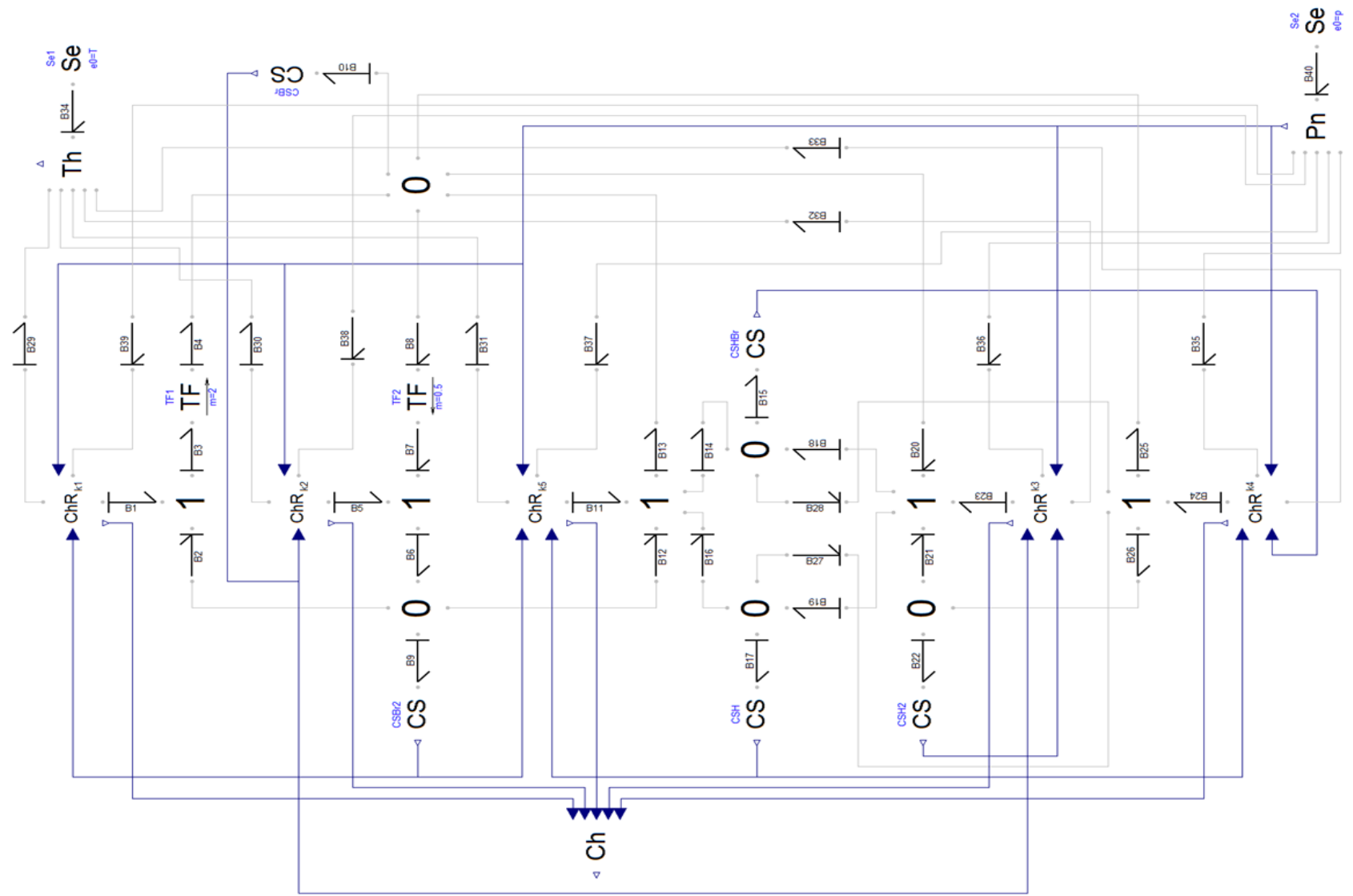

Fig.2: Completed chemical reaction bond graph 
Initially, we start out with $0.0075 \mathrm{~mol}$ of $\mathrm{H}_{2}$ and $\mathrm{Br}_{2}$ each, create and consume the temporary atoms $\mathrm{Br}^{\circ}$ and $\mathrm{H}^{\circ}$, and after roughly $0.07 \mathrm{~s}$, we end up with $0.015 \mathrm{~mol}$ of $\mathrm{HBr}$.

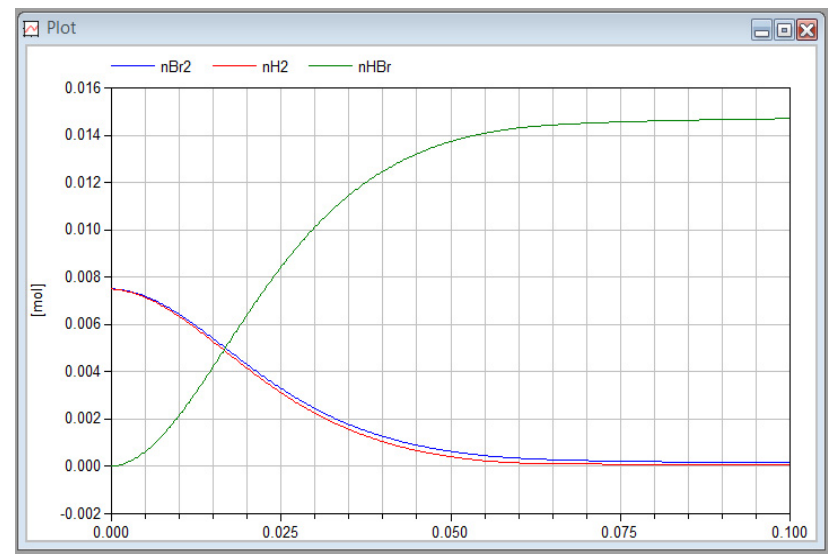

Fig.3: Chemical reaction trajectories

The same results could have been obtained more easily by writing down the reaction rate equations directly, but the presented bond graph model is superior, because it represents not only the dynamics of the mass flows through the reactions, but also the corresponding energy flows. Each chemical bond contains two variables, an effort $\mu$, representing a chemical potential, and a molar flow rate $v$, representing the mass flow, such that:

$$
P=\mu \cdot v
$$

is the power flowing through the bond.

Hence the bond graph can also be used to compute the power flows through the reaction network, and we notice that the reaction is mildly exothermic, as documented in Fig.4, where the entropy gain is being shown. Roughly $\Delta S=0.06 \mathrm{~J} / \mathrm{K}$ of entropy are being generated during the reaction.

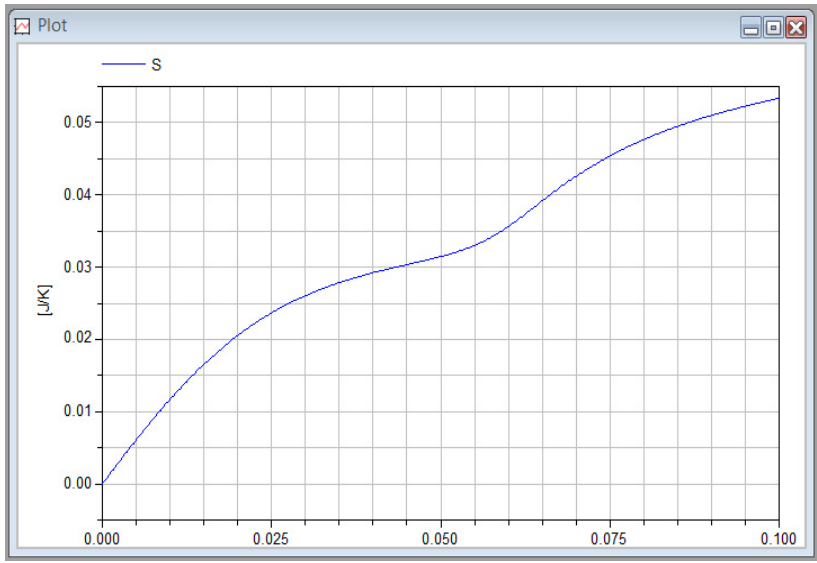

Fig.4: Entropy gain during chemical reaction
Unfortunately, the bond graph of Fig.2 is a mess, and few researchers will be willing to go through the agony of describing chemical reactions in this fashion. Therefore in the remainder of this paper, we shall improve on the model step by step, and create ever better models that can be used more easily.

\section{The Chemical Reaction Network}

The chemical potentials, $\mu_{i}$, of the reactants are computed by the CS-elements. These are then transferred across the chemical reaction network, which computes the chemical potentials of the individual step reactions, $\mu_{k i}$. The ChR-elements use that information to determine the molar flow rates of the step reactions, $v_{k i}$, which are then transferred back across the chemical reaction network, which computes the molar flow rates of the reactants, $v_{i}$. These are then integrated inside the CS-elements into the states, i.e., the number of moles, $n_{i}$, which in turn are needed by the ChR-elements to compute the flow rates. The state vector is transferred from the CS-elements back to the ChR-elements through separate signal paths, i.e., outside the bond graph.

As already demonstrated in [2], the chemical reaction network, relating reactants and step reactions to each other, can be interpreted as a multi-port transformer. Consequently, we can write:

$$
\begin{aligned}
&\left(\begin{array}{c}
\nu_{\mathrm{Br}_{2}} \\
\nu_{\mathrm{Br}} \bullet \\
\nu_{\mathrm{H}_{2}} \\
\nu_{\mathrm{H}} \\
\nu_{\mathrm{HBr}}
\end{array}\right)=\left(\begin{array}{rrrrr}
-1 & 1 & 0 & 0 & -1 \\
2 & -2 & -1 & 1 & 1 \\
0 & 0 & -1 & 1 & 0 \\
0 & 0 & 1 & -1 & -1 \\
0 & 0 & 1 & -1 & 1
\end{array}\right) \cdot\left(\begin{array}{c}
\nu_{k_{1}} \\
\nu_{k_{2}} \\
\nu_{k_{3}} \\
\nu_{k_{4}} \\
\nu_{k_{5}}
\end{array}\right) \\
&\left(\begin{array}{l}
\mu_{k_{1}} \\
\mu_{k_{2}} \\
\mu_{k_{3}} \\
\mu_{k_{4}} \\
\mu_{k_{5}}
\end{array}\right)=\left(\begin{array}{rrrrr}
-1 & 2 & 0 & 0 & 0 \\
1 & -2 & 0 & 0 & 0 \\
0 & -1 & -1 & 1 & 1 \\
0 & 1 & 1 & -1 & -1 \\
-1 & 1 & 0 & -1 & 1
\end{array}\right) \cdot\left(\begin{array}{c}
\mu_{\mathrm{Br}_{2}} \\
\mu_{\mathrm{Br}} \bullet \\
\mu_{\mathrm{H}} \\
\mu_{\mathrm{H}} \\
\mu_{\mathrm{HBr}}
\end{array}\right)
\end{aligned}
$$

This can also be written as:

$$
\vec{\nu}_{s}=\mathrm{N} \cdot \vec{\nu}_{r} \quad, \quad \vec{\mu}_{r}=\mathrm{M} \cdot \vec{\mu}_{s}
$$

where $\mathbf{N}$ is the transfer matrix for the flow rates, and $\mathbf{M}=\mathbf{N}^{\mathbf{T}}$ is the transfer matrix for the chemical potentials.

As we can describe the chemical reaction network using a multi-port transformer, it makes sense to extract the five CS-elements into a single CF-element, a capacitive field. As shown in Fig.5, this CFelement has on its left side, the five state variables, grouped into a state vector, whereas on the right side, 
there is now a vector of bond connectors, each representing one reactant.

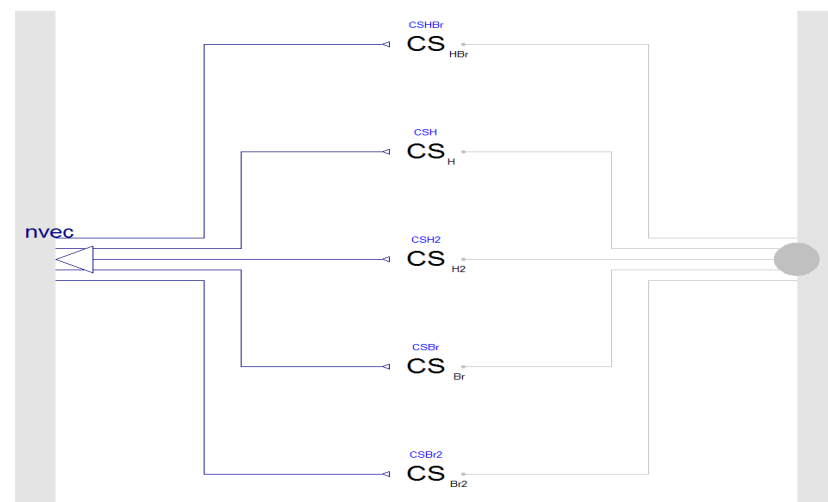

Fig.5: Capacitive field vector bond graph

For further processing, the vector of (grey) regular bond connectors needs to be converted to a single (blue) multi-bond connector, as shown in Fig.6. As the conversion element has been implemented as a bond, we need to add a 0 -junction also, in order to comply with the rule of all of our bond graph libraries that all macro elements must end in junctions, rather than in bonds.

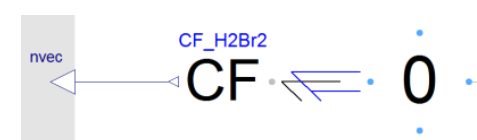

Fig.6: Conversion of bond vector to multi-bond

In the same way as with the five CS-Elements, we can also stack the five ChR-elements into a single RF-element, a resistive field, as shown in Fig.7.

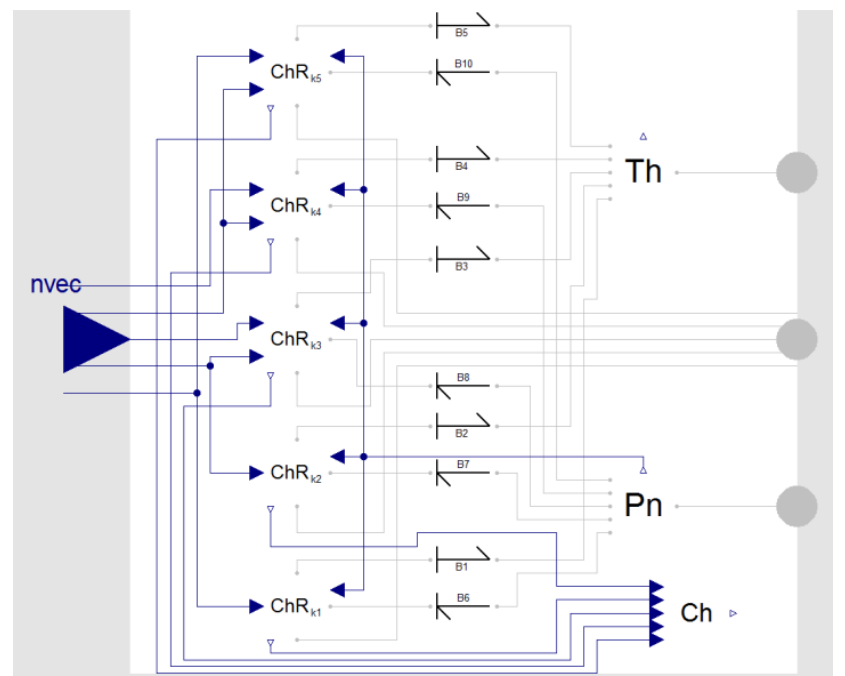

Fig.7: Resistive field vector bond graph

The attentive reader might already have discovered that the resistive field has three bond graph connectors: The one at the center on the right side is a vector connector representing the five mass flows as discussed before, whereas the bond graph connectors on top and below the mass flow connector are single connectors, one representing the heat flow and the other representing the volumetric flow. Under isothermal and isobaric operating conditions, these two connectors are used to impose from the outside the desired temperature of $T=800 \mathrm{~K}$ and the desired pressure of $p=102 \mathrm{kPa}$.

Finally, the vector connector of the RF-element also needs to be converted to a multi-bond connector, as shown in Fig.8.

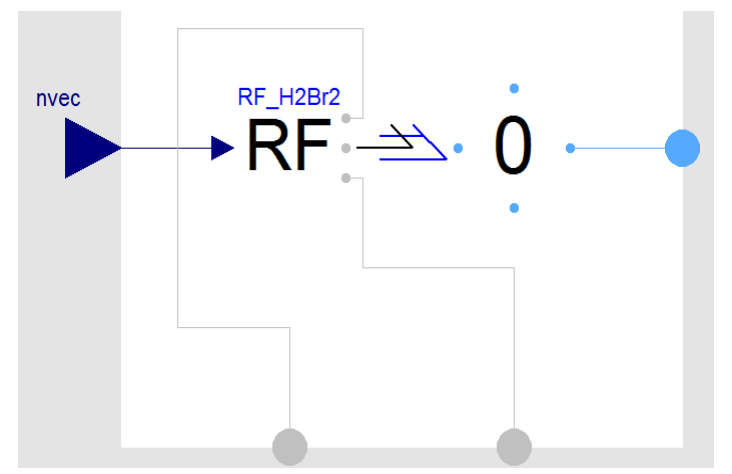

Fig.8: Conversion of bond vector to multi-bond

We are now ready to model the entire chemical reaction using MultiBondLib [6], a second bond graph library designed particularly for modeling mechanical systems in two and three space dimensions. Yet, the library can also be used for any other model that requires vectors of bonds.

The resulting model is shown in Fig.9. The chemical reaction network has not been modeled graphically in this model, but rather, was represented using a multi-port transformer ( $\left.\mathrm{TF}_{-} \mathrm{H} 2 \mathrm{Br} 2\right)$. The unconnected MBG defaults model shown at the bottom right corner of Fig.9 represents the multi-bond world model that sets the default dimension of all multibonds to a value of five.

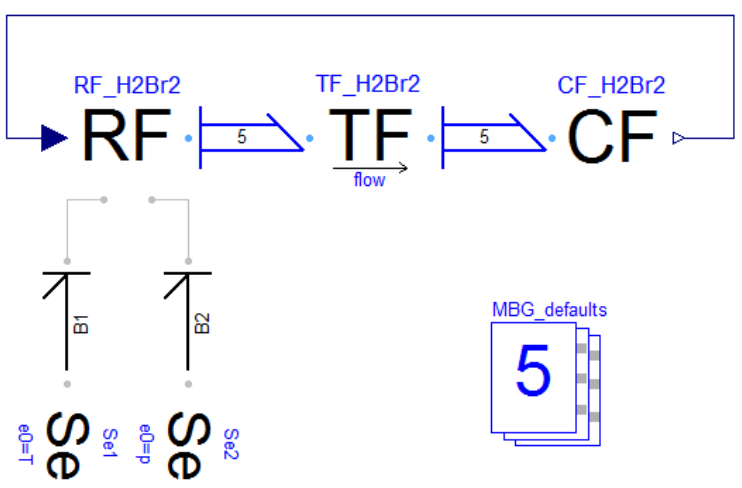

Fig.9: Chemical reaction modeled using multi-bonds

The simulation results obtained with this code are exactly the same as with the model of Fig.2, as these are truly identical models. 


\section{Thermo-bonds and Chemo-bonds}

As shown in Fig.10, an excerpt of Fig.1, each ChRelement has three bond graph connectors, one representing mass flow (variables $\mu$ and $v$, i.e., chemical potential and molar flow rate), a second representing volumetric flow (variables $p$ and $q$, i.e., pressure and volumetric flow rate), and a third representing heat flow (variables $T$ and $S d o t$, i.e., temperature and entropy flow rate). Each of the three pairs, when multiplied, represents a power flow measured in Watts.

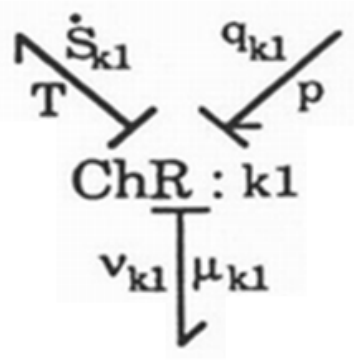

Fig.10: ChR-element

Although the three bonds represent different physical phenomena, they are mathematically identical. For this reason, it is important that bond variables are declared as 'Real' in Modelica, rather than be associated with particular measurement units. These variables may inherit measurement units through the higher layers of the model architecture, but by themselves, bond graph variables are neutral.

It is not possible to have mass flows without accompanying volume flows and heat flows, because the masses always carry their own volume and heat with them. The internal energy of matter of a mass can be written as:

$$
U=T \cdot S-p \cdot V+\mu \cdot n
$$

with the corresponding power flow:

$$
U d o t=T \cdot S d o t-p \cdot q+\mu \cdot v
$$

Thus, each mass flow can be interpreted as a parallel connection of three individual power flows, one representing the mass flow itself, a second representing the accompanying volumetric flow, and a third representing the heat flow.

As these three flows belong together, we have created a third bond graph library, called ThermoBondLib [3] that has been designed specifically for modeling convective flows.
Each thermo-bond represents a parallel connection of three regular bonds, one for each of the three types of power flow. This is shown in Fig.11.

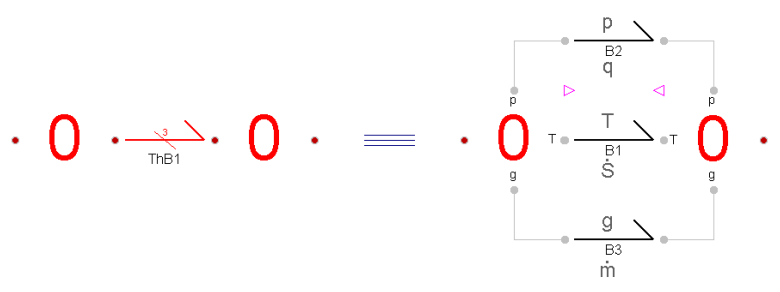

Fig.11: Composition of a thermo-bond

Since the thermo-bonds represent a specific physical phenomenon, it made sense to associate thermobonds with measurement units, i.e., whereas the regular (black) bonds of BondLib [4] and the (blue) multi-bonds of MultiBondLib [6] are neutral, the (red) thermo-bonds of ThermoBondLib [3] have been associated with measurement units explicitly.

For convective flows, it was convenient to measure the mass flow, $m d o t$, in $\mathrm{kg} / \mathrm{s}$. Consequently, the corresponding effort variable, $g$, must be measured in $\mathrm{J} / \mathrm{kg}$, such that their product is once again a power flow measured in Watts. The effort variable of mass flow, $g$, is the Gibbs potential, which sometimes is also called specific Gibbs energy.

Notice that $g$ has the same units as $h$, the specific enthalpy, but it is not the same quantity:

$$
h=g+T \cdot s
$$

where $s$ denotes the specific entropy, i.e., the entropy per unit mass.

Note: The Gibbs potential is still currently missing in the SIunits library of Modelica.

Since mass flows cannot occur without accompanying volume and heat flows, it makes sense to interpret chemical reactions as convective flows, and replace the (black) regular bonds of Fig.2 by (red) thermo-bonds. In this way, there will be no need any longer to treat the thermal and volumetric flows separately from the mass flows, and the ChR-elements will now only have one thermo-bond connector.

Unfortunately, it is inconvenient to represent mass flows in chemical reactions as absolute mass flows, measured in $\mathrm{kg} / \mathrm{s}$, because chemical reactions occur in relation to the number of molecules involved, and not in relation to the weight of the reactants. Correspondingly, chemical mass flows are given as molar flow rates, $v$, measured in $\mathrm{mol} / \mathrm{s}$, and correspondingly, the associated effort variable is the chemical potential, $\mu$, measured in $\mathrm{J} / \mathrm{mol}$.

It is easy to convert between the two types of mass flows. This is simply a transformation, as 
shown in Fig.12. It would have been possible to deal with molar flow rates using the 'redeclare' feature of Modelica, but it was simpler to create yet another bond graph library, ChemBondLib, this time featuring green chemo-bonds, that are identical to the red thermo-bonds except for the way in which the mass flows are being represented.

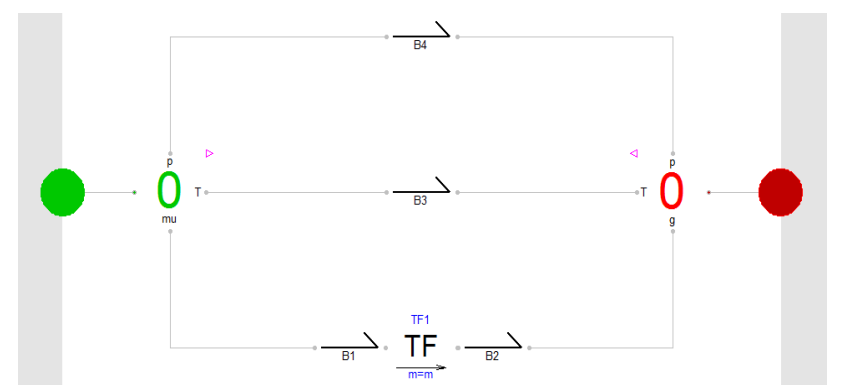

Fig.12: Conversion from absolute mass flow rates to molar flow rates

We are now ready to formulate the third version of the chemical reaction model. This new model is shown in Fig.13.

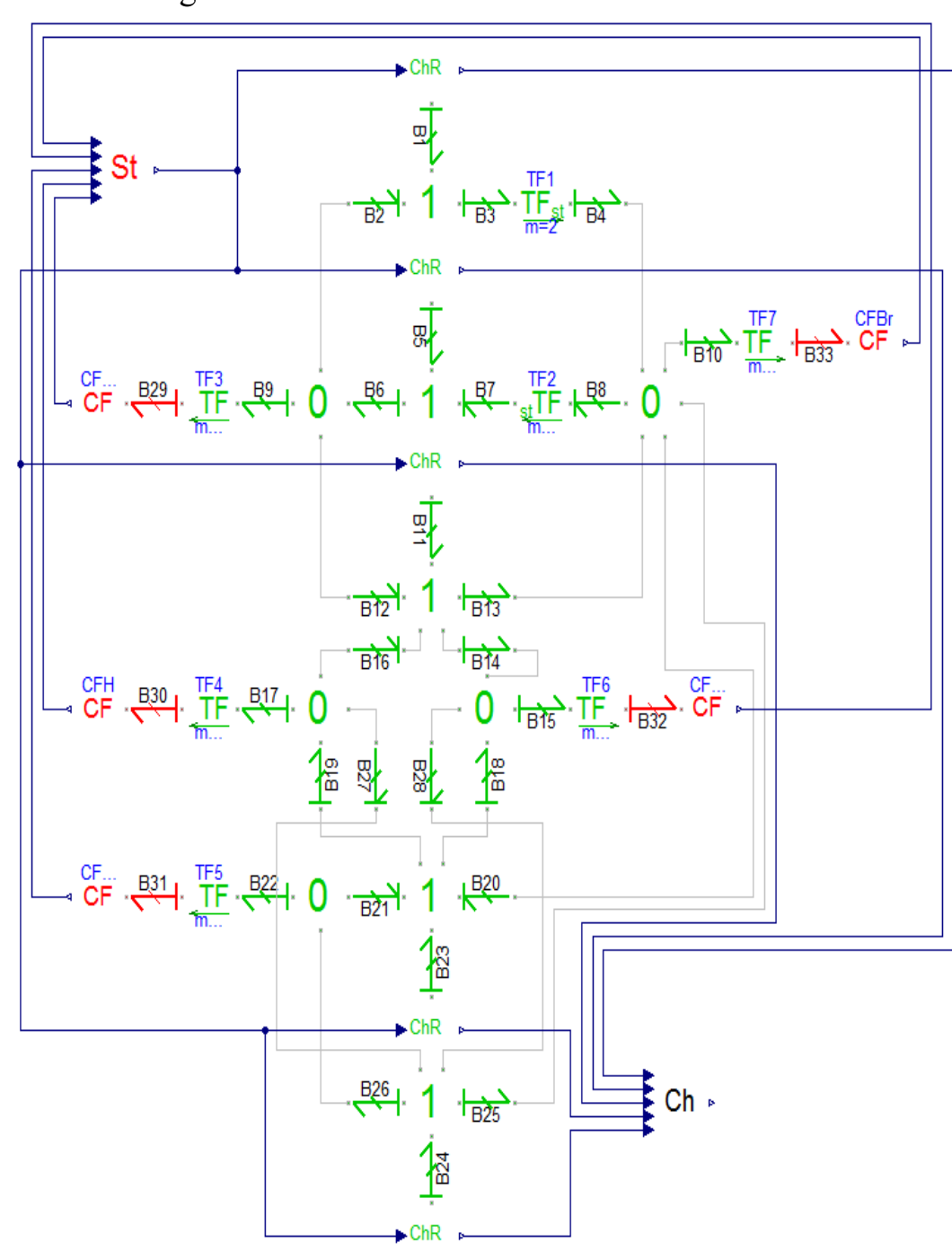

The entire chemical reaction network is now shown in green. Each of the green bonds models three parallel energy flows. As the substances, represented through the (red) CF-elements, are modeled using absolute masses (they are the very same CF-elements that we had introduced in [3] before), one of the transformers shown in Fig.12 is placed between the (green) chemical reaction network and each of the (red) CF-elements.

The model is much simpler than that of Fig.2, because there is no longer any need to deal with the heat and volumetric flows separately. These flows are now transferred across the reaction network together with the mass flows.

The CF-elements now compute the three potential (effort) variables, $T, p$, and $\mu$. These variables are then transferred across the chemical reaction network to the side of the step reactions. The ChR-elements compute the three flow variables, Sdot, $q$, and $v$. These are transferred back across the reaction network to the side of the reactants.

Each CF-element computes a partial state vector including the three variables $S, V$, and $n$. The partial state vectors are concatenated to a complete state vector in the St-model that transfers the state information back to the ChRelements, as the reactor models require the state information to compute the flows.

As expected, the simulation results obtained by this third version of the model are identical to those received earlier.

\section{Multi-chemo-bonds or Chemo-multi-bonds ?}

We can now once again eliminate the graphical representation of the chemical reaction network and replace it by a multi-port transformer.

To this end, we again stack the five CF-elements, as shown in Fig.14.

On the right side, we now have a vector of (green) chemo-bond connectors, each representing three flows, a heat flow, a volumetric flow, and a molar mass flow. Thus, the vector chemo-bond connector can be interpreted as a multi-chemo-bond connector.

Fig.13: Chemical reaction modeled by chemo-bonds 


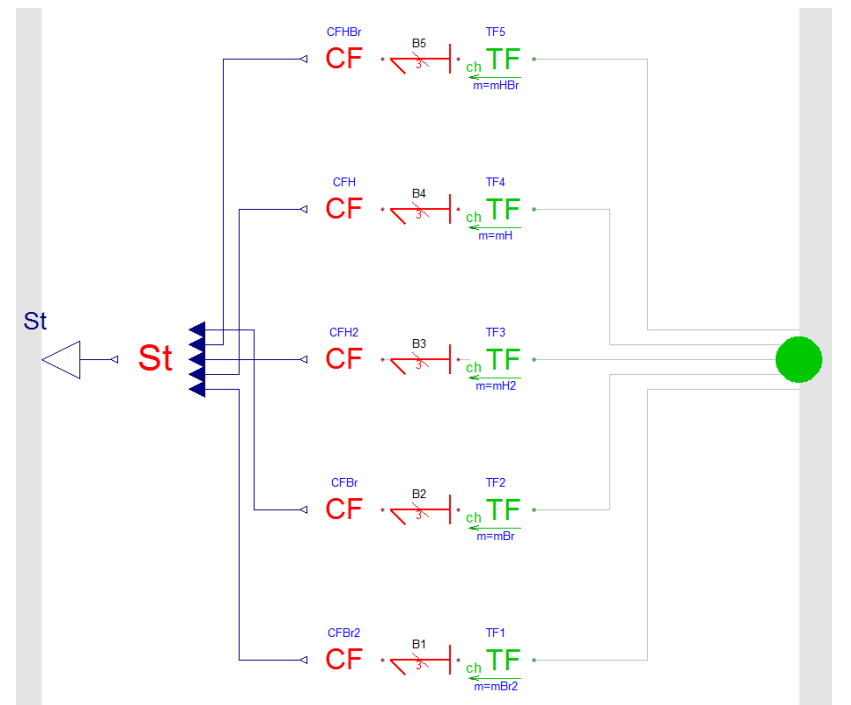

Fig.14: Capacitive reactant field

Fig. 15 shows, how the vector of individual chemo-bonds gets converted to a single multi-bond. Here, the multi-bond connector represents a vector of length 15.

However for reasons that will become clear very soon, it was more convenient to rearrange the sequence of bonds within the multi-bond in such a way that the multi-bond contains first the five heat flows, followed by the five volumetric flows, followed by the five molar mass flows. Thus, the multi-bond can be interpreted as a chemo-multi-bond connector.

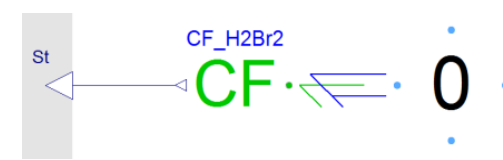

Fig.15: Conversion of connectors

In the same fashion, we can also stack the five ChRelements, as shown in Fig.16, with the corresponding conversion of connectors shown in Fig.17.

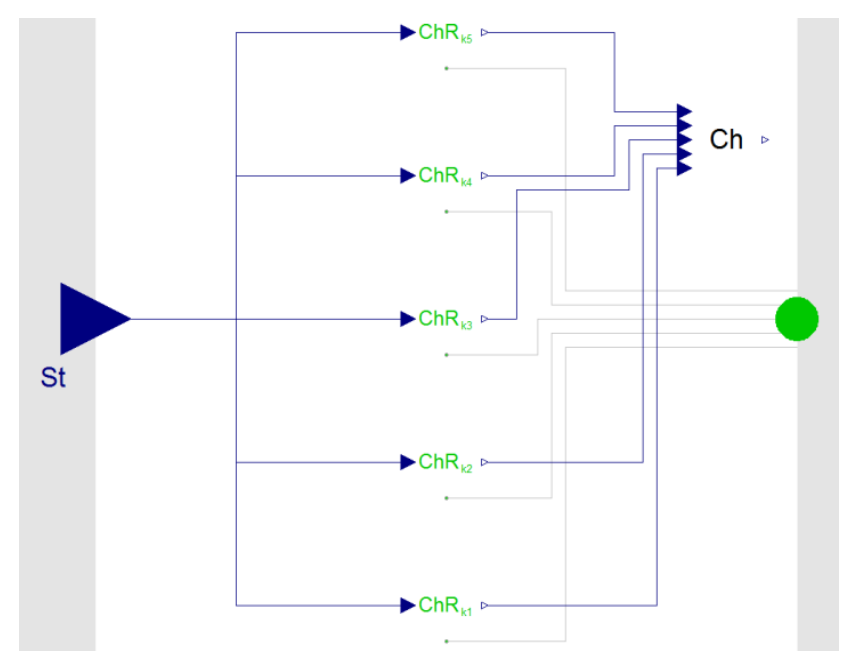

Fig.16: Resistive reaction field

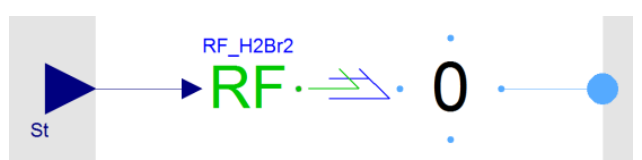

Fig.17: Conversion of connectors

As expected, the stacked reactor model of Fig.16 is much simpler than the corresponding stacked reactor model of Fig.7, because the thermal and pneumatic flows are not handled separately any longer.

The complete model (fourth version) is shown in Fig.18.

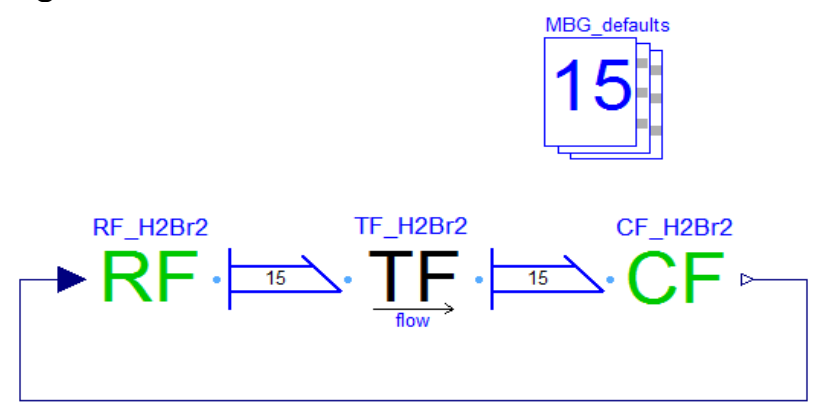

Fig.18: Chemical reaction modeled by multi-chemobonds

This time around, the transformation matrix of the multi-port transformer is of size $15 \times 15$. Yet, it can be composed easily from the previously introduced $\mathbf{N}$-matrix, assuming that we operate on multi-chemobonds rather than chemo-multi-bonds:

$$
\begin{aligned}
& \mathbf{Z}=\operatorname{zeros}(5,5) \\
& \mathbf{M}=\left(\begin{array}{lll}
\mathbf{N} & \mathbf{Z} & \mathbf{Z} \\
\mathbf{Z} & \mathbf{N} & \mathbf{Z} \\
\mathbf{Z} & \mathbf{Z} & \mathbf{N}
\end{array}\right)
\end{aligned}
$$

The transformation matrix is a block-diagonal matrix containing the same $\mathbf{N}$-matrix three times along the diagonal, once used to transform the heat flows, once used to transform the volumetric flows, and once used to transform the molar mass flows.

A comparison of the computational efficiencies of the four models is given in Table 1 .

Table 1: Comparison of computational efficiency

\begin{tabular}{|c|r|r|r|}
\hline Model & \# original & \multicolumn{1}{|c|}{ \# state } & \# algebraic \\
\hline Version & equations & variables & \multicolumn{1}{c|}{ variables } \\
\hline $\mathbf{1}$ & 1195 & 8 & 57 \\
\hline $\mathbf{2}$ & 1187 & 8 & 55 \\
\hline $\mathbf{3}$ & 3507 & 16 & 72 \\
\hline $\mathbf{4}$ & 2835 & 16 & 66 \\
\hline
\end{tabular}


The first two models are indistinguishable concerning their computational efficiencies. The third and fourth model are a bit less efficient computationally. The reason is the following. In the first two models, we recognized that we could add up the partial entropy flows and the partial volume flows and integrate them together, whereas in models three and four, each CF-element contains three separate states, one describing the partial entropy, a second describing the partial volume, and a third describing the partial mass of each of the five reactants.

\section{Conclusions}

In this paper, we have shown how different types of bond graphs can be used to describe chemical reaction systems.

Only a single reaction system was used for demonstration, namely a hydrogen-bromine reaction simulated under isothermal and isobaric operating conditions.

Although we have been able to demonstrate that the models become simpler as we treat chemical reactions as convective flows, there is a yet much more important reason for doing this, a reason that does not become obvious from reading this paper alone.

Amrhein simulated the same reaction system under a series of different operating conditions [1,2]. He replaced the isothermal condition by an adiabatic condition, for example, and he replaced the isobaric condition by an isochoric condition.

It becomes clear from reading his documents that each of his models looks slightly different, i.e., the bond graph had to be adjusted a bit from one set of operating conditions to another.

The reason for this inconvenience is that Amrhein did not compute all quantities where they naturally belong. Consequently, his models violated some of the premises of object-oriented modeling. All of the potential (effort) variables and all of the state variables should be computed at the side of the reactants, whereas all of the flow variables should be evaluated at the side of the step reactions.

Using the convective flow approach to modeling chemical reaction systems, it won't be necessary any longer to modify the bond graph from one set of operating conditions to another, because all variables are indeed being computed where they belong.

The implementation of the (red) CF-elements used in the simulations of this paper are not yet fully general. As we were only interested in isothermal and isobaric operating conditions, it sufficed to set the temperature, $T$, and the pressure, $p$, to their de- sired values inside the CF-elements. Since both the temperature and the pressure were assumed constant, also the chemical potentials, $\mu_{i}$, are constant, and consequently, also those values could be entered as parameters.

In the general case, $T, p$, and $\mu_{i}$ will not be constant, and they will have to be computed from state information, as already demonstrated in the general CF-elements for air and water presented in [3].

Temperature and pressure equilibration between different reactants can be modeled using heat exchange (HE) elements and pressure/volume exchange (PVE) elements, placed between the different CF-elements, as demonstrated in [3].

In the special case of an isothermal operating condition, a controlled entropy flow out of the storages (CF-elements) could then be added to the model that equals the entropy flow generated by the reaction system, such that the total entropy, and thereby also the temperature remain constant. Alternatively, an HE-element could be added between the reaction system and the environment that guarantees that the temperature of the reaction system remains at the ambient temperature.

In the special case of an isobaric operating condition, either a controlled volume flow would need to be imposed from the outside, or alternatively, a PVEelement could be added between the reaction system and the environment that guarantees that the pressure of the reaction system remains at the ambient pressure.

Thus, general CF-elements can be used independently of the operating conditions, which then are imposed on the model by external control flows in the same way as a chemical engineer would set up his or her experiment in the lab.

Unfortunately, we did not have either the time or the space in this paper to demonstrate how such a setup would need to be modeled using the ChemBondLib and ThermoBondLib Modelica libraries, and therefore, the proposed generalization will have to be postponed to another time and another publication.

The research presented in this paper represents only a very first step in an ongoing research effort. Reactions among ideal gases are particularly easy to model and simulate, and isothermal and isobaric operating conditions are among the most convenient operating conditions that may be assumed.

Much more research is needed before we can claim that we have created a universal approach to modeling and simulating all kinds of chemical reaction systems in a truly object-oriented physically inspired manner. 


\section{References}

[1] Amrhein, M.: Bond Graph Modeling of Chemical Reaction Dynamics. MS Thesis, Department of Electrical and Computer Engineering, University of Arizona, Tucson, AZ, 1990

[2] Cellier, F.E.: Continuous System Modeling. Springer-Verlag, New York, 1991

[3] Cellier, F.E. and Greifeneder, J.: ThermoBondLib - A New Modelica Library for Modeling Convective Flows, In: Proc. $6^{\text {th }}$ International Modelica Conference, Bielefeld, Germany (2008) Vol.1, 163-172

[4] Cellier, F.E. and Nebot, A.: The Modelica Bond Graph Library, In: Proc. $4^{\text {th }}$ International Modelica Conference, Hamburg, Germany (2005) Vol.1, 57-65

[5] Oster, G.F., Perelson, A.S., and KatzirKatchalsky A.: Network Thermodynamics: Dynamic Modelling of Biophysical Systems, In: Quarterly Reviews of Biophysics (1973) Vol. 6(1), 1-134

[6] Zimmer, D. and Cellier, F.E.: The Modelica Multi-bond Graph Library, In: Proc. $5^{\text {th }}$ International Modelica Conference, Vienna, Austria (2006) Vol.2, 559-568

\section{Author Biographies}

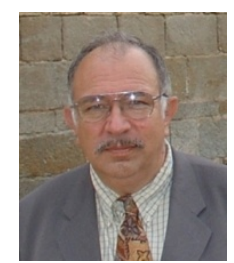

François E. Cellier received his BS degree in electrical engineering in 1972, his MS degree in automatic control in 1973, and his $\mathrm{PhD}$ degree in technical sciences in 1979, all from the Swiss Federal Institute of Technology (ETH) Zurich. Dr. Cellier worked at the University of Arizona as professor of Electrical and Computer Engineering from 1984 until 2005. He then returned to his home country of Switzerland. Dr. Cellier's main scientific interests concern modeling and simulation methodologies, and the design of advanced software systems for simulation, computer aided modeling, and computer-aided design. Dr. Cellier has authored or co-authored more than 200 technical publications, and he has edited several books. He published a textbook on Continuous System Modeling in 1991 and a second textbook on Continuous System Simulation in 2006, both with Springer-Verlag, New York.

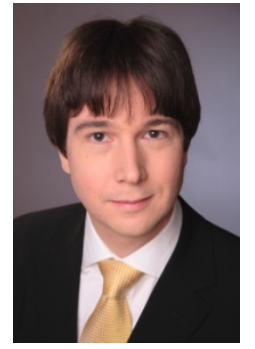

Jürgen Greifeneder received a diploma degree in Engineering $\mathrm{Cy}-$ bernetics from the University of Stuttgart, Germany in 2002. He then switched to the University of Kaiserslautern, where he received a Ph.D. in Electrical and Computer Engineering in 2007 with a dissertation on the formal analysis of temporal behavior of Networked Automation Systems (NAS) by use of probabilistic model checking. Scientific stays at the University of Arizona (USA), at the Ecole Normale Supérieure de Cachan (F), and at the Universidade de Brasília (BR) completed his education. Since 2008, Dr. Greifeneder is with the ABB Corporate Research Center, Ladenburg, Germany, working in the area of automation engineering with a focus on simulation-based engineering. Dr. Greifeneder's primary research interests concern modeling and simulation methodologies. 